\title{
A Lamb Wave Lens for Acoustic Microscopy
}

\author{
Abdullah Atalar, Senior Member, IEEE, Hayrettin Köymen, Senior Member, IEEE, and F. Levent Değertekin
}

\begin{abstract}
In a conventional scanning acoustic microscope the excited leaky modes contributes significantly to the high contrast obtained in the images. However, all such modes exist simultaneously, and the interpretation of the images is not straightforward, especially in layered media. A new lens geometry is proposed that can be used with acoustic microscopes to image layered solid structures. This new lens can focus the acoustic waves in only one of the Lamb wave modes of the layered solid with a high efficiency. $V(Z)$ curves obtained from this lens are more sensitive to material properties compared to that obtained from conventional lens. Measuring the return signal as a function of frequency results in another characteristic curve, $V(f)$. The Lamb wave lens and the associated characterization methods for the layered structures are described. The results presented show that the Lamb wave lens is at least an order of magnitude more sensitive than the conventional lens and can differentiate between a good bond and a disbond in a layered structure easily.
\end{abstract}

\section{INTRODUCTION}

A COUSTIC MICROSCOPE is a powerful instrument for the quantitative characterization of materials [1]. In particular, important structural parameters like film adhesion [2], elastic constants and residual stresses [3] on layered solids can be measured. Subsurface images of such structures can also be obtained using the excited leaky layer waves [4]. Leaky layer waves such as leaky-Rayleigh waves or generalized Lamb waves can be excited in a planar layered structure immersed in liquid by bulk waves incident at the surface at some critical angles [5]. The critical angles are determined mainly by layer thicknesses, elastic properties of the layer/substrate materials and the frequency of operation. When a scanning acoustic microscope is used to image a layered solid structure, almost all possible acoustic wave modes are excited in the structure, because the acoustic microscope lens creates all incidence angles at the object interface. In this case, the images are cluttered by the presence of many simultaneous modes. Some of these excited modes are bulk waves, and some are leaky modes that eventually return to the liquid medium. The efficiency of excitation is rather low, because an appreciable part of input power is wasted at angles where there is no subsurface excitation. An interference of specularly reflected rays with leaky modes gives rise to the well-known $V(Z)$ effect that is responsible for the high contrast in the acoustic images. When used with a layered structure, the presence of many modes makes the interpretation of $V(Z)$ rather difficult. From such images one hopes to detect flaws

Manuscript received April 15, 1992; revised and accepted July 14, 1992. This work was supported by the Turkish Scientific and Technical Research Council, TUBITAK.

The authors are with the Department of Electrical and Electronics Engineering, Bilkent University, Ankara, Turkey 06533.

IEEE Log Number 9203243 like delaminations, disbonds, layers under stress, changes in elastic parameters, etc. Although there exists methods [6] of recovering elastic parameters of the material under test from the received signal, the results of these computations are not very accurate and most of the time the inversion problem is ill-conditioned.

We propose a different lens design that produces images easy to interpret. The new Lamb wave lens resembles a conventional acoustic microscope lens except that it employs a conical focusing surface rather than a spherical one. It can excite subsurface acoustic waves with a high efficiency. As the critical angle of a layered material depends on frequency [7], the Lamb wave lens can selectively excite the leaky modes by matching the fixed incidence angle with the corresponding critical angle at a particular frequency. $V(Z)$ obtained with this new lens, is formed by interference of a single leaky mode and the specular reflection providing a direct and very sensitive measurement technique for the leaky wave velocity of the object. Recording the received signal amplitude, $V$, as a function of varying frequency, $f$, one obtains a unique $V(f)$ curve that is highly dependent on elastic parameters of the layer, of the substrate and the quality of the bonding. This provides a characterization method for layered structures. The $V(f)$ method was proposed earlier [8] to be used with conventional lens. That combination, however, was not suitable for characterizing layered structures. $V(f)$ technique has some advantages over the $V(Z)$ method: A fast frequency scan is sufficient to get $V(f)$ while a slow mechanical scanning is needed for $V(Z)$. Moreover, the interpretation of $V(f)$ is easier than that of $V(Z)$.

In this paper, we first give a theoretical analysis of the Lamb wave lens based on angular spectrum approach and ray acoustics. We find an expression that relates the signal output to the reflection coefficient of the object, and to the geometrical parameters of the lens. To show the subsurface characterization potential of the system, layers with perfect bonding, disbond and delamination are investigated. We find that the new lens differentiates between these interfaces with a high sensitivity. For experimental confirmation, we use a Lamb wave lens and present measured $V(Z)$ and $V(f)$ curves for layered materials along with calculated results.

\section{EXCiTATion OF LEAKY MODES}

A layered solid immersed in liquid supports leaky modes known as Rayleigh-like (LR) waves [9] and generalized Lamb waves [10]. The LR wave is like a Rayleigh wave and it is confined to the surface. This wave is dispersive, and the dispersion is determined by the wavelength and the thickness of the layer(s). The generalized Lamb wave modes are like 


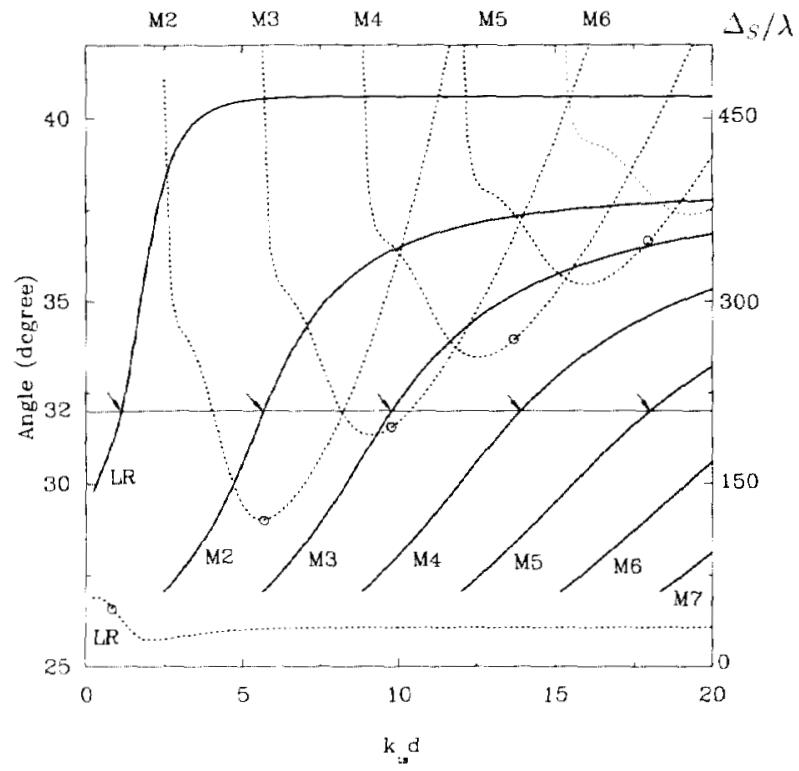

Fig. 1. Excitation angle from liquid side (solid) and Schoch displacement of various modes in wavelength units (dotted) as a function of $k_{t s} d$ for a copper layer on steel.

Lamb wave modes in a plate and they are also dispersive. When the layered solid is immersed in liquid, all these modes become leaky, as their energy is radiated into the liquid medium. In this case, it is also possible to excite these modes by a bulk wave insonification in the liquid medium [7]. Corresponding to each mode there exists a critical angle of incidence [11]. Since the modes are dispersive, the critical angles depend on the frequency. The dispersion curves can be obtained by examining the reflection coefficient at the liquidlayered solid boundary. At the critical angles the reflection coefficient has phase transitions.

Here, we consider solids containing only one layer. We calculated the dispersion characteristics for a number of structures using the reflection coefficient. Fig. 1 shows the dispersion curves of leaky-Rayleigh wave and the Lamb wave modes for a copper layer on a steel substrate. The horizontal axis is the wavenumber-thickness product, $k_{t s} d$. Here, $k_{t s}$ is the shear wavenumber of the substrate and $d$ is the thickness of the layer. As opposed to the common convention, the vertical axis is the critical angle of incidence. For small $k_{t s} d$ values only the LR mode exists. For $k_{t s} d=0$, the critical angle, $\theta$, is equal to the Rayleigh critical angle of the substrate. As $k_{t s} d$ increases, the critical angle changes and approaches to the Rayleigh critical angle of the layer material. The first Lamb wave mode (M2) is excited only when $k_{t s} d$ is greater than a threshold value. For greater $k_{t s} d$ values, the critical angle varies toward the shear critical angle of the layer material. Higher order Lamb wave modes (M3, M4, etc.) appear at even greater values of $k_{t s} d$. At the cut-off point, the critical angle is equal to the shear critical angle of the substrate material.

A wedge transducer [12] can be used to excite these modes efficiently from the liquid side. An efficiency measure of such a transducer for a particular mode can be deduced from the variation of the reflection coefficient phase at the critical angle. In particular, the slope of the phase variation is an important parameter [13]. This slope is proportional to the Schoch displacement, $\Delta_{S}$. The optimum detection efficiency between two equal wedges is about $66 \%$, when the wedge width, $W$, is [14] $W=0.628 \Delta_{S} \cos \theta$, where $\theta$ is the critical angle. This efficiency figure ignores the angular spread of the beam due to finite aperture, and it will be lower when that effect is included. In Fig. 1 we plot $\Delta_{S} / \lambda$ with respect to $k_{t s} d$ for the same solid structure where $\lambda$ is the wavelength in the liquid. For LR mode, $\Delta_{S} / \lambda$ is the lowest, while the same value for the Lamb wave modes exhibit a minimum for $k_{t s} d$ values very close to their respective cut-off values. For a practical wedge width, very large $\Delta_{S} / \lambda$ values must be avoided. Therefore, for efficient Lamb wave excitation, the minimum points of these curves must be targeted. The $k_{t s} d$ and $\Delta_{S} / \lambda$ combinations of a wedge angle resulting an incidence angle of $32^{\circ}$ is also depicted in Fig. 1. It is interesting that the $k_{t s} d$ value at which each mode is excited, is also quite close to the minimum $\Delta_{S} / \lambda$ value for that particular mode. Hence it is possible to choose a wedge angle that will excite most of the lower modes quite efficiently.

\section{LAMB WAVE LENS}

The foregoing discussion outlines the conditions for efficiently coupling the Lamb wave modes in a layered structure by a wedge transducer. A wedge transducer is not suitable to use in an imaging system due to its poor resolution. A method of focusing Lamb waves was described earlier [14]. That system utilized a cylindrical reflecting surface and a wedge transducer. However, its resolution was not very good because of the limited convergence angle. Here, we describe a new configuration with a better resolution. The basic idea is to use a full conical wave rather than a section of it. The creation of a conical wave can be achieved either by a conical transducer [15] or by refraction from a suitable conical surface. The geometry of the proposed Lamb wave lens is illustrated in Fig. 2. A conical recess replaces the spherical cavity of the conventional lens. The acoustic waves produced by the transducer will first hit the conical refracting surface before they reach the object surface. An antireflection layer exists on the conical surface to reduce mismatch loss. It can be easily proved that all the refracted rays from the conical surface are incident at the object surface at the same angle. If the inclination of generated conic waves is chosen to be a critical angle for a mode of Lamb waves, a large fraction of the energy will be converted to a leaky Lamb wave mode. The excited evanescent Lamb waves converge and focus at the intersection of the cone axis with the object surface. The focused waves then diverge and leak back into the liquid medium. Upon refraction from the conical surface, they are detected by the transducer. If an inhomogeneity is present at the focus point, the received electrical signal will be disturbed and will diminish in amplitude. Such a lens will have an axial resolution equal to the thickness of the layer, since the Lamb wave modes exist predominantly in the layer. Since the f-number of the lens is very small and no aberrations are involved, it is possible to obtain lateral resolutions better than a wavelength. 


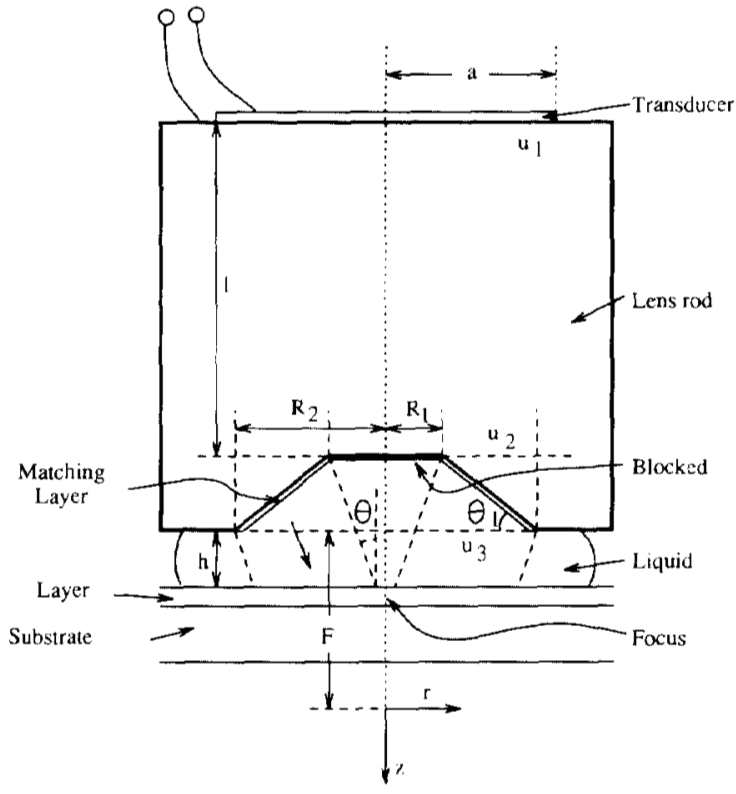

Fig. 2. Geometry of the Lamb wave lens.

If the distance, $h$, between the object surface and the lens is close to $F$, dominantly the specularly reflected rays are received by the transducer and a large signal amplitude will be obtained. As $h$ is reduced the signal due to specular reflection will get smaller. For $h<\left(R_{1} / \tan \theta-\left(R_{2}-R_{1}\right) \tan \theta_{1}\right)$ no specular rays will be received. In this range, only leaky waves will contribute. Obviously one obtains a signal at the transducer terminal that is entirely due to leaky waves, if the central part is blocked. The lens performance and hence the received signal is more sensitive to material and interface properties, however, when the central part is not blocked and leaky waves are allowed to interfere with the specular reflection passing through this part.

One apparent problem of the lens geometry is that the incidence angle of rays at the object surface is fixed and this angle may not coincide with a critical angle for Lamb waves for another layer thickness. This problem can be overcome easily by adjusting the excitation frequency. Since the Lamb wave modes are dispersive, the critical angle of a Lamb wave mode can be made equal to the fixed angle of the Lamb wave lens at the proper frequency. The experimental determination of frequency is very easy: one needs to tune the frequency until the maximum signal is received.

Since the bandwidth of most transducers are not very wide, for some samples it may be impossible to hit a critical angle within the tuning range. Therefore, one needs a series of Lamb wave lenses with different cone angles to be able to cover all possible samples. The design of the Lamb wave lens involves the following considerations. The cone angle must be decided using the dispersion curves for the layered material or materials of interest. The transducer size and the rod length must be chosen to minimize the diffraction loss and the interference from spurious pulses. For good efficiency the lens aperture must be of suitable size. The transducer bandwidth must be wide enough to allow a frequency scan.

\section{Response of the Lamb Wave Lens}

The amplitude of the received signal can be found by an application of the angular spectrum technique. For simplicity we assume a circularly symmetric geometry. Consider the geometry shown in Fig. 2. We assume a circular transducer of radius $a$ placed a distance $l$ away from the conical refracting surface. A planar object surface is placed a distance $h$ away from the refracting surface as shown. We write the scalar potential, $u_{1}^{+}$, at the plane of the transducer as

$$
\begin{aligned}
u_{1}^{+}(x, y) & =A \operatorname{circ}\left[\left(x^{2}+y^{2}\right)^{1 / 2} / a\right] \text { where } \\
\operatorname{circ}(r) & =\left\{\begin{array}{ll}
1 & r \leq 1 \\
0 & r>1
\end{array} .\right.
\end{aligned}
$$

The angular spectrum, $U_{1}^{+}$, at the same plane is given by a jinc function:

$$
\begin{aligned}
U_{1}^{+}\left(k_{x}, k_{y}\right) & =\mathcal{F}\left\{u_{1}^{+}\right\}=4 a^{2} A \operatorname{jinc}\left[(a / \pi)\left(k_{x}^{2}+k_{y}^{2}\right)^{1 / 2}\right] \\
& =2 \pi a^{2} A J_{1}\left[a\left(k_{x}^{2}+k_{y}^{2}\right)^{1 / 2}\right] / a\left(k_{x}^{2}+k_{y}^{2}\right)^{1 / 2}
\end{aligned}
$$

where $J_{1}$ is the Bessel function of the first kind. The spectrum, $U_{2}^{+}$, at $z=l$ is written as

$$
U_{2}^{+}\left(k_{x}, k_{y}\right)=4 a^{2} A \operatorname{jinc}\left[(a / \pi)\left(k_{x}^{2}+k_{y}^{2}\right)^{1 / 2}\right] \exp \left(j k_{z} l\right) .
$$

The scalar potential, $u_{2}^{+}$, at the plane of the conical lens can be found by an inverse Fourier transformation.

$$
u_{2}^{+}(x, y)=\mathcal{F}^{-1}\left\{U_{2}^{+}\left(k_{x}, k_{y}\right)\right\} .
$$

Due to the limited aperture of the conical surface, only the part of the field distribution for which $R_{1} \leq\left(x^{2}+y^{2}\right)^{1 / 2} \leq R_{2}$ is significant. At this point we use the ray theory to find the field at the surface of the conical refractor surface. We keep the amplitude of the field fixed, but change its phase according to the path length for each ray. Then, we use the ray theory again to take care of refraction effect through the Snell's Law. Referring to Fig. 3 , the field at the plane $3, u_{3}^{+}$, is approximately

$$
u_{3}^{+}(r)=\left\{\begin{array}{lc}
\left(r^{\prime} / r\right)^{1 / 2} A_{R} u_{2}^{+}\left(r^{\prime}\right) \exp \left\{j\left(k_{1} d_{1}+k_{2} d_{2}\right)\right\} \\
R_{1}<r^{\prime}<R_{2} \\
0 & \text { elsewhere }
\end{array}\right.
$$

where

$$
A_{R}=\left(1+\tan \theta_{1} \tan \left(\theta_{1}-\theta_{2}\right)\right)^{1 / 2}
$$

$r^{\prime}=\left(r+R_{2} \tan \theta_{1} \tan \left(\theta_{1}-\theta_{2}\right)\right) /\left(1+\tan \theta_{1} \tan \left(\theta_{1}-\theta_{2}\right)\right)$

$$
k_{1} \sin \theta_{1}=k_{2} \sin \theta_{2}
$$

$d_{1}=\left(r^{\prime}-R_{1}\right) \tan \theta_{1}$ and $d_{2}=\left(R_{2}-r^{\prime}\right) \tan \theta_{1} / \cos \left(\theta_{1}-\theta_{2}\right)$.

where $k_{1}$ and $k_{2}$ are the wavenumbers in the lens rod and in the liquid medium, respectively. Notice that, the diffraction in the short distance between the planes 2 and 3 is neglected. At this point, the geometrical theory of diffraction could be used, but it is not essential for accurate results, since the output 


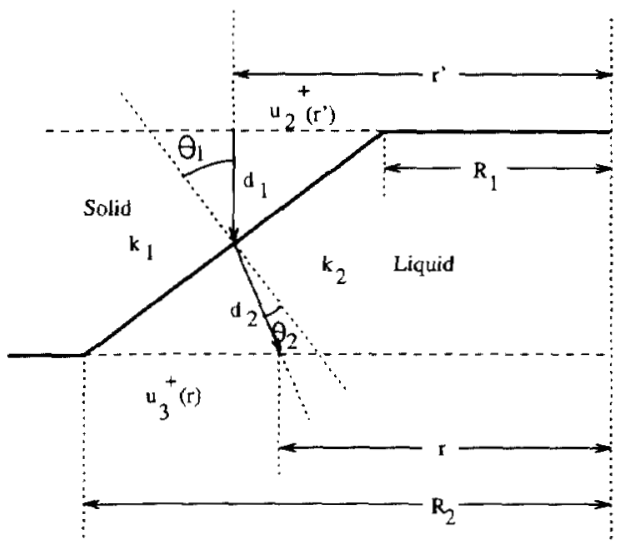

Fig. 3. Geometry for the ray theory.

voltage is obtained by integrating the field. At plane 3 we can transform into the angular spectrum domain to take care of the propagation in $z$ direction and the reflection process at the object interface. The reflected angular spectrum at the same plane, $U_{3}^{+}$, is given by

$$
U_{3}^{-}\left(k_{x}, k_{y}\right)=U_{3}^{+}\left(k_{x}, k_{y}\right) \exp \left\{j 2 k_{z}^{\prime} h\right\} \mathcal{R}\left(k_{x} / k_{2}, k_{y} / k_{2}\right)
$$

where

$$
U_{3}^{+}\left(k_{x}, k_{y}\right)=\mathcal{F}\left\{u_{3}^{+}(x, y)\right\} \text { and } k_{z}^{\prime}=\left(k_{2}^{2}-k_{x}^{2}-k_{y}^{2}\right)^{1 / 2} \text {. }
$$

The exponential factor above takes care of a total propagation distance of $2 h$ and the reflection process at the surface of the layered structure is included through a multiplication by the reflection coefficient, $\mathcal{R}\left(k_{r} / k_{2}\right)$. The voltage output of the transducer due to reflected field can be found from

$$
V=K \int_{-\infty}^{+\infty} \int_{-\infty}^{+\infty} k_{z}^{\prime} U_{3}^{+}\left(-k_{x},-k_{y}\right) U_{3}^{-}\left(k_{x}, k_{y}\right) d k_{x} d k_{y}
$$

Since the problem has circular symmetry we can make the substitution

$$
k_{r}=\left(k_{x}^{2}+k_{y}^{2}\right)^{1 / 2}
$$

where $k_{r}$ is radial component of the wave number and $K$ is a constant. Then, we write the output voltage, $V$, as a function of excitation frequency, $f$, and the position of object with respect to geometrical focal plane, $Z=h-F$, as

$$
V(f, Z)=2 \pi K \int_{0}^{+\infty} k_{r} k_{z}^{\prime} U_{3}^{+}\left(k_{r}\right) U_{3}^{-}\left(k_{r}\right) d k_{r} .
$$

Here, the $V(f, Z)$ is expressed in terms of lens geometry, the reflection coefficient of the object material, operation frequency and object separation. The evaluation of $V$ at a single point involves two Fourier transformations and an integration operation. Note that, the expression above does not include the frequency response of the transducer.

The result obtained applies when the central part of the lens is blocked. If the central part is not blocked, then there will be an additional term, due to specular reflection.

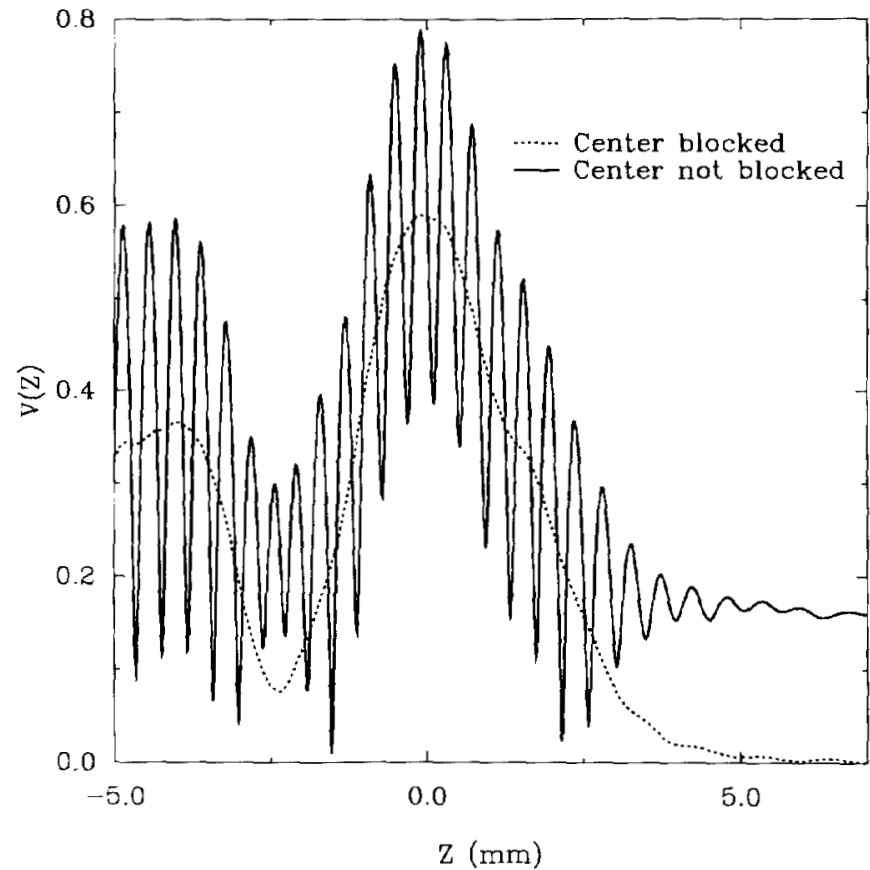

Fig. 4. Lamb wave lens $V^{-}(Z)$ curves (center blocked and unblocked) for 0.6-mm copper on steel $(f=9.6 \mathrm{MHz})$. The lens is made of aluminum, with $a=8.5 \mathrm{~mm}, l=5.9 \mathrm{~cm}, R_{1}=3.1 \mathrm{~mm}, R_{2}=5.95 \mathrm{~mm}$ and $\theta_{1}=45.5^{\circ}$.

\section{THE $V(Z)$ CURVES}

$V(Z)$ measurement resulting with a center blocked Lamb wave lens gives an interference free curve (dotted curve in Fig. 4). The peak at $Z=0$ is due to specularly reflected rays. The second peak around $Z=-4 \mathrm{~mm}$ is created by the leaky waves. The vertical axis of the graph is normalized so that $V(0)=1$ for a perfect reflector. The relative magnitude of the second peak indicates that the overall detection efficiency for leaky waves is about $14 \%$. If the energy lost in the blocked center is ignored, the efficiency rises to $27 \%$. This figure is less than what can be attained with an optimal Lamb wave lens geometry.

If the waves incident from the central part of the Lamb wave lens is allowed to overlap the leaky waves, an interference mechanism similar to the conventional lens results. For this purpose the center of the lens should not be blocked, in which case a $V(Z)$ curve with a good number of fringes is easily obtained. In Fig. 5, calculated $V(Z)$ curve for a layered structure composed of $0.6-\mathrm{mm}$ copper on steel substrate is depicted together with measurements. Here, the frequency of operation is $7.8 \mathrm{MHz}$ and there is a disbond at the interface. For large positive $Z$ values, no interference is visible. There, the only contribution is from the normal specular component. At points near $Z=0$ the interference results from the superposition of normal specular component and the oblique specular reflection. The period of the fringes is determined by the cone angle of the Lamb wave lens, and it carries no or little information about the object. For more negative $Z$ values $(Z<-2.5) \mathrm{mm}$, however, the interference is between normal specularly reflected rays and the leaky waves. In this case, the periodicity is determined by the leaky wave velocity of the particular mode. The agreement of measurements with 


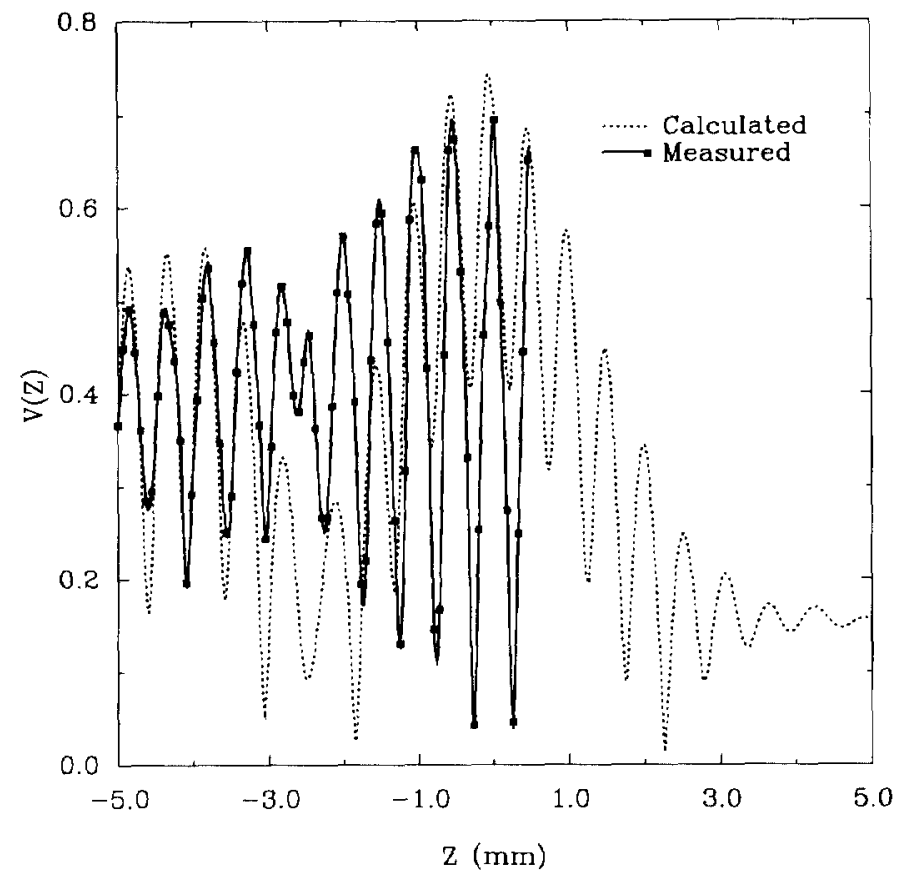

Fig. 5. Measured and calculated Lamb wave lens $V(Z)$ curve for $0.6-\mathrm{mm}$ copper layer on steel with disbond $(f=7.8 \mathrm{MHz})$.

calculated $V(Z)$ is remarkably good. The discrepancy in amplitude is due to the simplifying assumption in calculations that the normal specular component is proportional to the relative area at the center. The periodicity in $V(Z)$ curves is the principal property that constitutes sensitivity. Fig. 6 depicts the $V(Z)$ variation for negative $Z$ values for a layered material when the $c_{44}$ of the layer is perturbed by $1 \%$. Clearly, $0.5 \%$ perturbation in the shear wave velocity is easily detectable. With $40 \mathrm{~dB}$ signal to noise ratio and a perfect mechanical scanning system, it is possible to detect a velocity perturbation of $0.0075 \%$ in the layer material. With such a perturbation, the maximum difference between the two $V(Z)$ curves is $1 / 100$ (corresponding to $40 \mathrm{~dB}$ ) of the peak value. Under the same assumptions a conventional lens with an envelope detector can only detect a $0.12 \%$ velocity perturbation. Obviously, it is possible to obtain better sensitivity [16] with a higher signal-to-noise ratio or with signal processing techniques.

\section{VI. $V(f)$ CuRves}

As the frequency is scanned while $Z$ is kept constant, a characteristic $V(f)$ curve is traced where the peaks signify the existence of modes. The $V(f)$ curve is highly dependent on the phase transitions of the reflection coefficient. Since, any perturbation of elastic or physical parameters such as the thickness, density or elastic constants of the layer causes a shift in the position of the phase transitions, a shift in the positions of $V(f)$ peaks is also expected.

The material property discrimination ability of the Lamb wave lens with a blocked center was previously investigated by means of simulation [17]. It was shown that a 5\% change in $c_{44}$ of the layer material causes nearly $5 \%$ shift in the peak positions. Similarly, a reduction in layer density, $\rho$, causes a shift in the same direction. Corresponding sensitivity figure

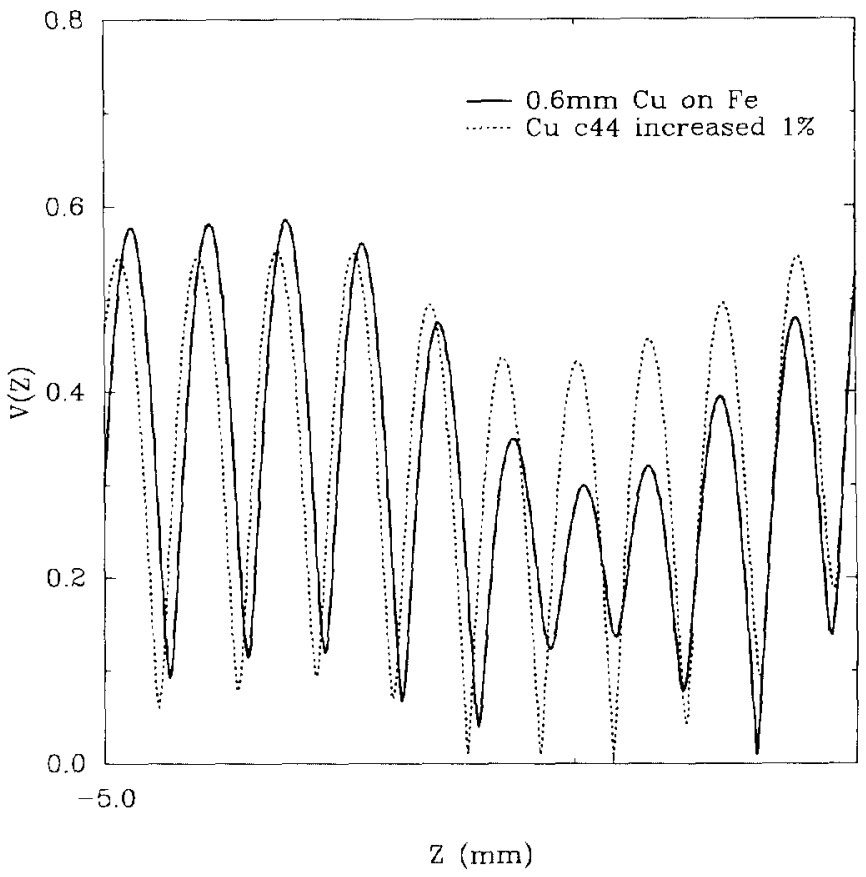

Fig. 6. Lamb wave lens $V(\mathcal{Z})$ curves for copper on steel (solid) and for copper layer $c_{44}$ perturbed by $1 \%$ (dashed) $(f=9.6 \mathrm{MHz})$.

for the layer density is slightly higher. The layer $c_{11}$ and substrate parameters are found to have negligible effect. The shear velocity of the layer material has the greatest influence.

The sensitivity of $V(f)$ curve with respect to material properties can be significantly improved by allowing the specularly reflecting signal from the central part to interfere with the leaky signal. This $V(f)$ is depicted in Fig. 7 for 0.6 mm copper layer on steel sample. One can observe a fringe pattern imposed on the envelope whose morphology is the same as $V(f)$ obtained in the center blocked case. This fringe pattern is very sensitive to the elastic parameters of the layers and the bond quality at the interfaces.

Sensitivity of $V(f)$ to material properties and the type of interface is tested on two test phantoms using a lens with unblocked central part. The phantoms are made of copper layers on steel obtained by electroplating. One of the phantoms contained a region of disbond and the other contained a region of delamination, while good adhesion is maintained elsewhere on the samples. In order to have a strong adhesion to substrate, steel surface must be treated by copper cyanide prior to plating in copper sulphate solution. In order to induce a poor bond, a region is masked during copper cyanide solution. Then, the mask is removed and plating is continued in copper sulphate solution. Both a disbond and a delamination are obtained using this method. In Fig. $8, V(f)$ for good bond and delamination is compared for a copper layer of $0.5 \mathrm{~mm}$, both numerically and experimentally. Note that precise measurement of thickness of the layer is also possible through matching the fringes. Fringes are particularly pronounced for good bond at frequencies around $6.5 \mathrm{MHz}$, whereas for delamination that frequency is $8.5 \mathrm{MHz}$. In Fig. 9 , good bond is compared to disbond. $V(f)$ curve very clearly differentiates between the two bonds. The agreement 


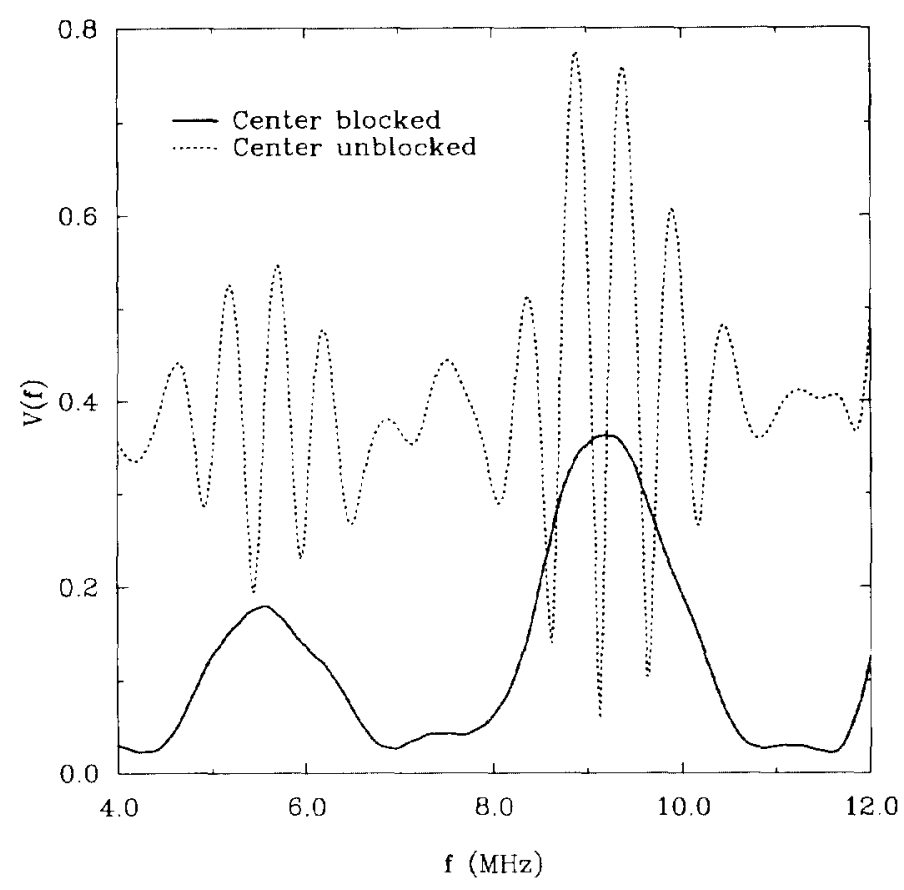

Fig. 7. Calculated $I(f)$ curves for 0.6 -mm copper layer on steel in case of good bond using lenses with blocked and unblocked central part.

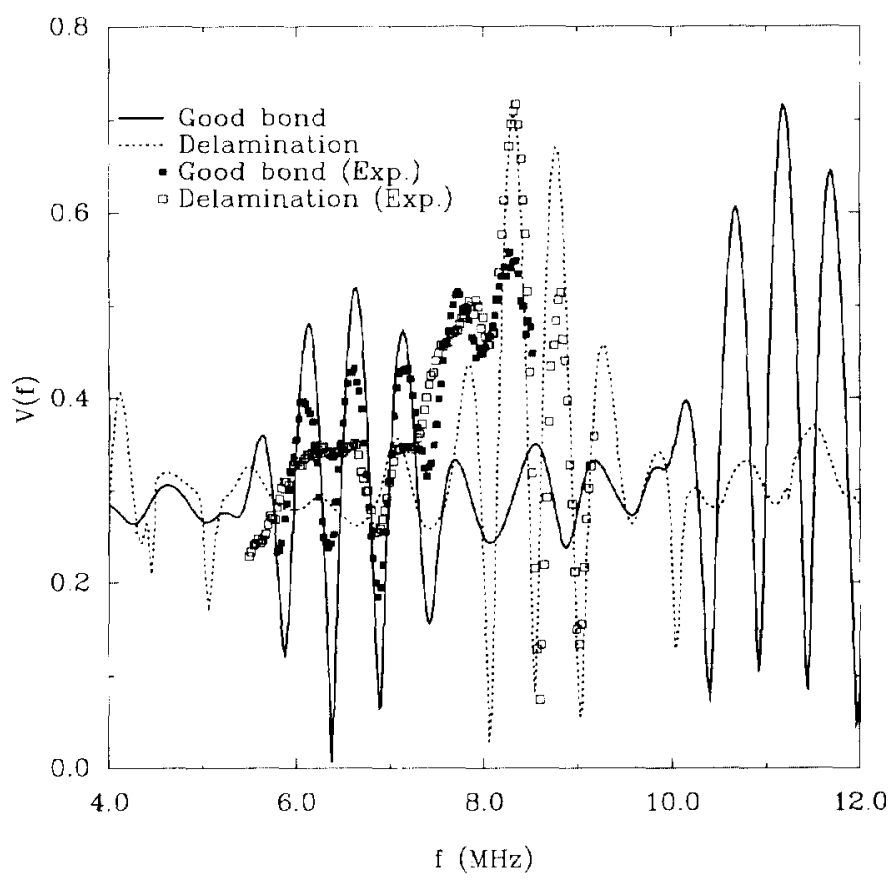

Fig. 8. Calculated and measured $L^{\prime}(f)$ curves for $0.5-\mathrm{mm}$ copper layer on steel with good bond and delamination using a lens with unblocked central part.

of experimental results with simulation is particularly good where the interference induced fringe amplitude is high. Most probably, lack of parallelism between the lens aperture and the sample surface is responsible for the slight mismatch in case of good bond in Fig. 9. In calculations, the disbond is simulated by a slippery boundary condition at the layersubstrate interface, whereas a thin layer of air is assumed to exist between the layer and substrate in case of delamination.

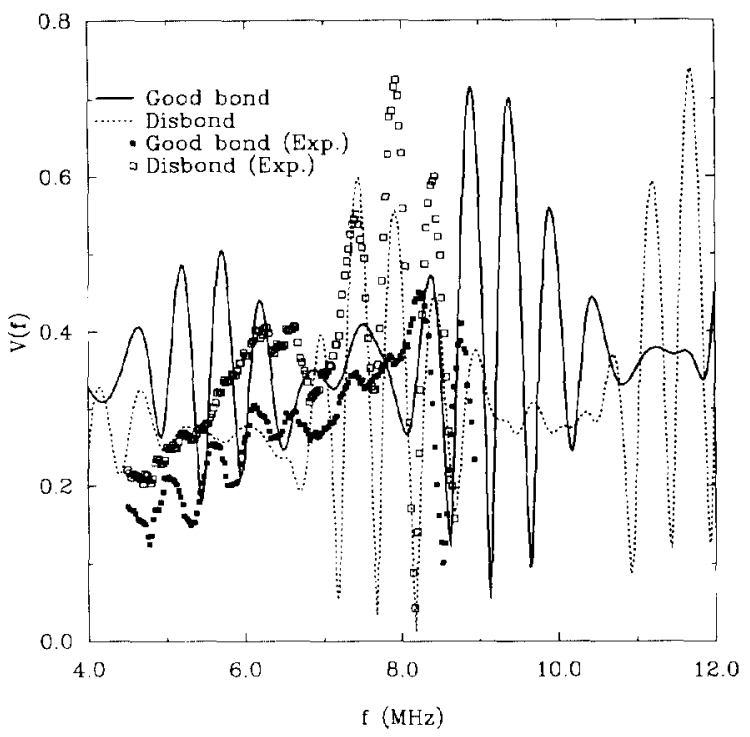

Fig. 9. Calculated and measured $V(f)$ curves for 0.6- $\mathrm{mm}$ copper layer on steel with good bond and disbond using a lens with unblocked central part.

All the presented curves are obtained by normalizing the transducer output by the amplitude of the normal reflection signal from the central part. Since the normalization is done both on measured and calculated data, the frequency response of the transducer is eliminated.

Inversion of $V(Z)$ [6] of the conventional acoustic microscope results in the reflection coefficient that in turn gives information about the critical angles. To be able to perform the $V(Z)$ inversion operation, the phase of the received signal is necessary, but it is difficult to obtain. On the other hand, the critical angle information is available from the $V(f)$ curves directly without a need for inversion. The $V(f)$ curves result in peaks at positions corresponding to different Lamb wave modes. The positions of the peaks are most affected by the nature of the bond (good-bond/disbond/delamination) and by the shear elastic constant and density of the layer. It can be shown via simulation of $V(f)$ curves at $40 \mathrm{~dB}$ signal to noise ratio, it is possible to detect $0.01 \%$ perturbation in shear wave velocity of the layer material.

\section{CONCLUSION}

The Lamb wave lens can complement the conventional lens in acoustic microscopes for some applications due to its inherent ability to focus waves in a subsurface layer with a high efficiency. The new lens is suited to image layered structures with little lateral variation. To use such a lens, the acoustic microscope must have the ability to vary its operation frequency. The frequency must be tuned to excite a suitable Lamb wave mode. This new lens does not have a critical focal plane as the spherical lenses have. The only requirement is to keep the distance to the object below a certain limit. However, the parallelism of the lens aperture and the object surface is critically important.

$V(Z)$ characterization using this lens is more sensitive to that achievable by spherical lenses. Typically, the improvement in sensitivity is more than an order of magnitude. In this 
paper, a new and very sensitive material property and interface measurement method for layered materials is also proposed. The method involves producing a $V(f)$ curve using Lamb wave lens for the material under investigation, which provides the dispersion characteristics of the supported modes. This measurement is simple and fast, requiring only a frequency scan.

The Lamb wave lens is completely compatible with the existing manufacturing technology of the conventional acoustic lenses. Because of the simplicity of manufacturing a conical surface, it is plausible to build Lamb wave lenses with very small diameters. For such small lenses the path that must be traveled in the highly lossy liquid medium is quite small, enabling the focusing system to work at very high frequencies, possibly at frequencies not achievable with the conventional acoustic microscope lens.

The described lens geometry is not sensitive to the anisotropy of the object. A version of this lens sensitive to anisotropy can be constructed by making a straight groove instead of a conical recess. The cross section of the groove must be identical to that shown in Fig. 2. In this case, the leaky waves will propagate in only one direction in the anisotropic object. The relation of this lens to the conical geometry is analogous to the relation of the line-focus-beam acoustic microscope lens to the spherical lens. Also utilization of a shear wave transducer in a lens with present geometry may increase sensitivity to anisotropy, as suggested with a spherical lens [18].

\section{REFERENCES}

[1] P. A. Reinholdtsen, C-H. Chou, and B. T. Khuri-Yakub, "Quantitative acoustic microscopy using amplitude and phase imaging," in Proc. IEEE Ultrason. Symp. 1987, pp. 807-811.

[2] B. Derby, G. A. D. Briggs, and E. R. Wallach, "Non-destructive testing and acoustic microscopy of diffusion bonds," J. Mater. Sci., vol. 18, 1983.

[3] S. W. Meeks, D. Peter, D. Horne, K. Young, and V. Novotny, "Residual stress mapping with a scanning phase-measuring acoustic microscope," in Proc. IEEE Ulirason. Simp., 1989, pp. 809-812.

[4] G. C. Smith and M. G. Gee, "Observation of a sub-surface defect in sapphire by Rayleigh wave reflection in the scanning acoustic microscope," J. Mater. Sci. Lett., vol. 5, pp. 1133-1134, 1986.

[5] G. W. Farnell and C. K. Jen, "Excitation of acoustic beams in layered substrates," Rev. Phys. Appl., vol. 20, pp. 305-310, 1985.

[6] K. K. Liang, G. S. Kino, and B. T. Khuri-Yakub, "Material characterization by inversion of $\mathrm{I}(Z)$," IEEE Trans. Sonics Ultrason., vol. 32, pp. 213-224, 1985.

[7] D. B. Bogy and S. M. Gracewski, "Reflection coefficient for plane waves in a fluid incident on a layered elastic half-space," J. Appl. Mech., vol. 50, pp. $405-414,1983$.

[8] P. B. Nagy and L. Adler "Acoustic material signature from frequency analysis," J. Appl. Phys., vol. 67, pp. 3876-3878, 1990.

[9] D. E. Chimenti, A. H. Nayfeh, and D. L. Butler, "Leaky Rayleigh waves on a layered halfspace," J. Appl. Phys., vol. 53, pp. 170-176, 1982.

[10] D. E. Chimenti and A. H. Nayfeh, "Leaky Lamb waves in fibrous composite laminates," J. Appl. Phys., vol. 58, pp. 4531-4538, 1985.

[11] T. Kundu, "On the nonspecular reflection of bounded acoustic beams," J. Acoust. Soc. Am., vol. 83, pp. 18-24, 1988.

[12] H. L. Bertoni and T. Tamir, "Characteristics of wedge transducers for acoustic surface waves," IEEE Trans Sonics Ultrason., vol. 22, pp. $415-420,1975$

[13] A. H. Nayfeh and D. E. Chimenti. "Reflection of finite acoustic beams from loaded and stiffened half-spaces," J. Acoust. Soc. Am., vol. 75, pp. 1360-1368, 1984.

[14] A. Atalar and H. Köymen. "Use of a conical axicon as a surface acoustic wave focusing device," IEEE Trans. Ultrason., Ferroelec., Freg. Contr., vol. 34, pp. 53-63, 1987.
[15] S. Ayter, "Focusing surface waves using conical transducers," in Proc. IEEE Uitrason. Symp., 1987, pp. 301-304.

[16] J. Kushibiki. H. Takahashi, T. Kobayashi, and N. Chubachi, "Quantitative evaluation of elastic properties of $\mathrm{LiTa}_{3}$ crystals by line-focusbeam acoustic microscopy," Appl. Phys. Lett., vol. 58, pp. 893-895, 1991.

[17] A. Atalar, H. Köymen, and L. Değertekin. "Characterization of layered materials by the Lamb wave lens," in Proc. IEEE Ultrason. Symp., 1990, pp. $359-362$.

[18] C-H. Chou and B. T. Khuri-Yakub, "Acoustic microscopy with shear wave transducers," in Proc. IEEE Ultrason. Symp., 1987, pp. 813-816.

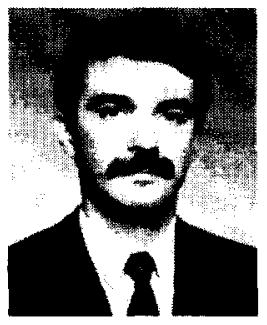

Abdullah Atalar (M'88-SM'90) was born in Gaziantep, Turkey, in 1954. He received the B.S. degree from Middle East Technical University, Ankara, Turkey, in 1974, and the M.S. and Ph.D. degrees from Stanford University, Stanford, CA. in 1976 and 1978, respectively, all in electrical engineering. His thesis work concerned reflection acoustic microscopy

From 1978 to 1980 , he was a Postdoctoral Fellow and later an Engineering Research Associate at Stanford University, continuing his work on acoustic microscopy. For eight months he was with Hewlett Packard Laboratories, Palo Alto, CA, where he engaged in photoacoustics research From 1980 to 1986, he was on the faculty of the Middle East Technical University as an Assistant Professor. While on leave from 1982 to 1983 , he worked at Ernst Leitz Wetzlar, Germany, where he was involved in the development of the commercial acoustic microscope. In 1986, he joined Bilkent University as the Chairman of the Department of Electrical and Electronics Engineering and founded the Department, where he is currently a Professor. His current research interests include acoustic imaging, linear acoustics. and computer-aided design in electrical engineering. He was awarded the H. Tuğaç Foundation Award of TUBiTAK. Turkey, for his contributions to acoustic microscopy.

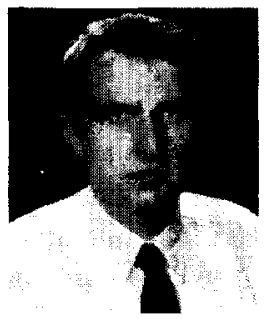

Hayrettin Köymen (S'74-M'76-M'87-SM'91) was born in Ankara, Turkey, on June 7, 1952 He received the B.Sc. and M.Sc. degrees from Middle East Technical University, Ankara, Turkey, in 1973 and 1976, respectively, and the Ph.D. degree from the University of Birmingham, Birmingham, England, in 1979, all in electrical engineering.

He became a faculty member of the Middle East Technical University in 1979. Until 1982 his work involved underwater acoustics and oceanographic instrumentation: since 1982, his work involved physiological signal acquisition and processing, medical ultrasonics and ultrasonic nondestructive evaluation. In 1990 , he joined the faculty of Bilkent University, Ankara, where he is now a Professor in the Department of Electrical and Electronics Engineering. His current research interests are personal computer-based biomedical signal acquisition and processing, medical imaging, finite amplitude effects in medical ultrasonics, and acoustic microscopy using leaky waves in layered media.

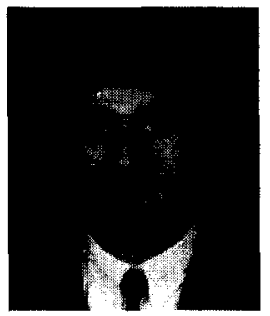

F. Levent Değertekin was born in Diyarbakır, Turkey, in 1968. He received the B.S. degree from the Middle East Technical University, Ankara, Turkey, and the M.S. degree from Bilkent University. Ankara. Turkey, in 1989 and 1991, respectively, both in electrical engineering. He is currently working toward the Ph.D. degree in electrical and electronics engineering at Bilkent University, where he has been a research assistant since 1989. His present research interests include acoustical imaging and nondestructive evaluation of layered materials. 